\title{
A case report of successful digoxin use for fast atrial fibrillation to facilitate emergency surgery in a bleeding patient
}

\author{
R Swart it \\ Department of Anaesthesiology, University of the Free State, South Africa \\ Corresponding author, email: reinier.swart@gmail.com
}

Atrial fibrillation complicated by a fast ventricular response rate can cause a significant physiological burden. This is characterised by systolic pump failure, decreased diastolic filling time and hypotension leading to impaired organ perfusion. Considering that most anaesthetic agents are cardiac suppressants, the additive effects of this condition together with anaesthesia may lead to increased perioperative morbidity and mortality. A 79-year-old male patient who presented with atrial fibrillation, caused by chronic obstructive pulmonary disease and pulmonary hypertension, and a fast ventricular response rate was booked for emergency ligation of the sphenopalatine artery for posterior epistaxis, complicated by significant blood loss. Both electrical and pharmacological alternatives exist to address this conundrum, but here we would like to present this case where digoxin was used successfully to rate control this patient perioperatively. Digoxin has recently fallen into disfavour for the chronic management of atrial fibrillation, and even in select cases of acute atrial fibrillation with a fast ventricular response rate, and the purpose of this case study is to highlight a potentially useful indication for this antiquated drug.

Keywords: acute anaesthesia, digoxin, atrial fibrillation (AF), rate control, emergency surgery

\section{Introduction}

Atrial fibrillation (AF) is defined by the American Heart Association (AHA) as "a supraventricular tachyarrhythmia with uncoordinated atrial activation and consequently ineffective atrial contraction."1 AF can occur in as many as $35 \%$ of patients over the age of 80 years. ${ }^{1}$ The perioperative implications of AF should not be underestimated, as a recent study showed that patients with AF preoperatively had an odds ratio of 1.69 (95\% confidence interval 1.34-2.14) for perioperative mortality, as compared to coronary artery disease, with a $6.4 \%$ incidence of 30-day mortality after non-cardiac surgery. ${ }^{2,3}$ Other morbidities associated with AF are inter alia heart failure, kidney impairment, atrial thrombus formation with stroke and other distant embolic phenomena. ${ }^{1}$

When the ventricles respond with a fast response rate to the atrial fibrillation (fast AF), diastolic filling time may be reduced, leading to lower preload, stroke volume and cardiac output. This may precipitate hypotension and systolic dysfunction of the left ventricle. ${ }^{4}$ The difficulty exists for anaesthetists in that many patients with decompensated physiology presenting for emergency surgery may exhibit fast $A F$, with resultant hypotension or clinical heart failure. Most anaesthetic drugs are also cardiac suppressants, which compound the problem for the perioperative patient presenting with fast AF.

Studies have proven the benefits of rate control over rhythm control in atrial fibrillation, especially if it is not new-onset atrial fibrillation. ${ }^{5}$ Many agents are promoted to achieve these ends, and in the acute setting a short-acting beta blocker is generally the first choice agent., ${ }^{1,6}$ Beta blockers have several side effects, though, such as bronchoconstriction, slowing of heart rate and suppression of inotropy, ${ }^{7}$ and beta blockers have proven to be dangerous in the setting of decompensated acute heart failure. ${ }^{8}$ Nondihydropyridine calcium channel blockers should also not be used in acute atrial fibrillation with depressed systolic function, as they may impair cardiac conduction and inotropy even further. Amiodarone is another agent that can be considered, but may take up to 7 hours to initiate rate control and has sympatholytic and atrioventricular nodal suppression properties, which make this agent hazardous in patients needing faster control with depressed systolic function. ${ }^{1}$ Digoxin, a cardiac glycoside, which acts by inhibiting the Na-K-ATPase pump, slows heart rate by lengthening the duration of the cardiac action potential and increasing inotropy. ${ }^{9}$ Digoxin may take up to an hour to take effect. ${ }^{1}$ Therefore, it does not have the benefit of faster onset of action that the beta blockers do, but has a different side effect profile. Although the AFFIRM trial showed increased mortality in patients taking chronic digoxin therapy, a propensitymatched post hoc analysis did not show increased mortality or hospitalisation in patients on chronic baseline digoxin therapy for paroxysmal or persistent atrial fibrillation., ${ }^{1,10}$ Other agents that can be considered for rate control are Vaughan Williams class $1 C$ antiarrhythmics, especially in patients with fast AF due to pre-excitation phenomena. ${ }^{1,11}$

\section{Case presentation}

A 79-year-old male was presented for emergent examination under anaesthesia and ligation of the sphenopalatine artery. The indication for surgery was acute posterior epistaxis. The patient was known to the otorhinolaryngology department with multiple previous episodes of epistaxis, and now presented with approximately one litre of measurable external blood loss.

Prior medical history revealed that he was a known hypertensive patient, controlled on enalapril $10 \mathrm{mg}$ twice daily and amlodipine $10 \mathrm{mg}$ daily. The patient was also known with chronic obstructive 
pulmonary disease (COPD) on home oxygen therapy - GOLD stage 1 - due to a protracted history of smoking (in excess of 20 pack years), which was complicated by pulmonary hypertension. The patient was also using budesonide and salbutamol metered dose inhalers for treatment of his COPD. He was anticoagulated with warfarin $5 \mathrm{mg}$ daily and acetylsalicylic acid $150 \mathrm{mg}$ daily for stroke prevention in atrial fibrillation (SPAF). Other medical history included benign prostatic hyperplasia, treated with tamsulosin $0.4 \mathrm{mg}$ daily, and gout, treated with allopurinol $300 \mathrm{mg}$ daily. He had a previous laparotomy for peptic ulcer disease and one previous examination under anaesthesia with no adverse anaesthetic outcomes. He did not have any significant family history of anaesthetic complications or comorbid conditions.

On examination the patient was alert but distressed and spitting small volumes of blood into a bucket which he held under his chin. The bucket contained an approximate volume of one litre of blood. He had a previous laparotomy scar and was edentulous. Further airway examination revealed a constant, but low volume stream of arterial blood down the back of his oropharynx. His cardiovascular status revealed an irregularly irregular tachyarrhythmia at a rate of 152 beats per minute (bpm) with a non-invasive blood pressure of 121/69.

Laboratory investigations revealed an international normalised ratio of 2.32, partial thromboplastin time of 33.2, normal kidney functions and electrolytes for age and his full blood count showed a haemoglobin level of 13.2 with a platelet count of 160 . Preoperative interventions initiated by the otorhinolaryngology team prior to presenting to theatre were the placement of a posterior nasal tamponade with a Foley's catheter, one gram of intravenous tranexamic acid and $10 \mathrm{mg}$ of intravenous vitamin $\mathrm{K}$.

On presenting to theatre, the blood bank was contacted to ascertain the availability of two units of packed red cells and one unit of platelets, and we were informed the cross-matching would require another 45 minutes to complete. This time was used to place an invasive arterial pressure monitoring line, another 18 gauge intravenous line and the patient was given a slow intravenous loading dose of $0.5 \mathrm{mg}$ digoxin. Freezedried plasma and prothrombin complex concentrate were also collected to be given in the event that bleeding on a capillary level could not be controlled. 50 minutes from arriving in the theatre reception area, two units of packed red cells arrived on a returnable basis. At this point the patient's heart rate had decreased to $127 \mathrm{bpm}$ and his blood pressure was 121/68, despite only receiving approximately 200 millilitres of lactated Ringer's solution up to this point.

A modified rapid sequence induction of anaesthesia in the semi-Fowler's position was achieved with $0.2 \mathrm{mg} / \mathrm{kg}$ etomidate and $1.3 \mathrm{mg} / \mathrm{kg}$ of succinylcholine after blunting the intubation response five minutes prior with a slow intravenous titration of fentanyl (up to $3 \mathrm{mcg} / \mathrm{kg}$ ). Intubation proceeded rapidly and without complication. The patient required vasopressor support after induction with a phenylephrine infusion (the maximum dose given was $1 \mathrm{mcg} / \mathrm{kg} /$ minute) while replacing intravascular volume with balanced crystalloids (one litre in total of Ringer's lactate solution). Endoscopic examination revealed a simple posterior epistaxis which ceased after arterial ligation and no significant capillary oozing was identified, therefore the decision was made to not risk thromboembolism by attempting to normalise the INR value any further. At the end of surgery, the patient's pulse was $87 \mathrm{bpm}$ with a blood pressure of 126/73. Arterial blood gas analysis at this point revealed a pH of 7.43 , $\mathrm{PaO} 2$ of $62, \mathrm{PaCO} 2$ of 34 , a base deficit of 1.5 , haemoglobin of 11.3 , lactate of 0.9 and the red blood cells were returned to the blood bank. The patient was extubated fully awake with blunting of the extubation response with intravenous lignocaine at $1 \mathrm{mg} / \mathrm{kg}$. No signs of ischaemia were noted on the ECG throughout the surgery.

\section{Discussion}

This case illustrates the role that intravenous digoxin may play in assisting with ventricular rate control in fast AF in patients presenting for urgent or emergent surgery with contraindications to beta blockers. The decision was made to avoid beta blockers, due to the aetiology for his atrial fibrillation being COPD. Beta blockers may have worsened his pulmonary hypertension and cardiac output (through increasing airway pressures). Amiodarone was also avoided as the onset of action would be longer than digoxin, with the possibility of hypotension in a bleeding patient. Nondihydropyridine calcium channel blockers are not readily available in intravenous formulation in our setting, and the possibility of haemodynamic collapse after initiating positive pressure ventilation in this patient was concerning. While the influence of volume loading and blunting the effect of anxiety on this patient's haemodynamics cannot be ignored as confounding factors, no untoward effects of digoxin were experienced and the patient's outcome was favourable. This patient was not discharged on chronic oral digoxin after cardiology review, likely due to the absence of signs of heart failure and the fact that the patient is an active individual. This is in keeping with the current guidelines, whereby chronic oral digoxin loses efficacy in patients who are still active. ${ }^{1}$

Digoxin does have several important adverse effects. When considering the perioperative administration of digoxin, one should remember that myocardial oxygen consumption may be increased, even in the presence of the non-failing, non-dilated heart, after the administration of the drug..$^{12}$ This patient did not show any signs of acute decompensation. Owing to its narrow therapeutic index it is important to implement therapeutic drug monitoring, especially in the elective perioperative setting. Acute toxicity is diagnosed by plasma drug levels above $2,5 \mathrm{ng} / \mathrm{ml}$ and may present as any dysrhythmia. ${ }^{13}$ Cardiac toxicity tends to present before other signs of toxicity, due to the predilection of digoxin for cardiac muscle. ${ }^{14}$ Typically a bradycardia is noted which may be of junctional origin. Acute toxicity is clinically detected by gastrointestinal features (nausea, vomiting, diarrhoea or abdominal pain), hyperkalaemia, bradyor tachydysrhythmias, lethargy, confusion and blurred or yellow vision. ${ }^{13}$ Acute toxicity (and acutely precipitated toxicity, as may 
be seen with intercurrent illness such as rapid changes in plasma potassium levels after vomiting in a patient on chronic oral digoxin) may be exacerbated by hypokalaemia, hypercalcaemia and hypomagnesaemia. ${ }^{7}$ When any of these factors are present, it is prudent to consider correcting these factors prior to administration of digoxin, as well as slower administration of the drug. No guidelines for changes in dosing could be found in the presence of hypovolaemia, however it is prudent to start with a smaller loading dose $(0.5 \mathrm{mg})$, as the lower volume of distribution may predispose the patient to acute toxicity, and repeat this dose if the desired effect has not been achieved yet.

Chronic digoxin toxicity is harder to diagnose and depends on a probabilistic diagnosis, which includes features of gastrointestinal symptomatology, bradycardia, cardiovascular automaticity and plasma digoxin levels. ${ }^{13}$ This is due to the higher skeletal muscle (up to 60 times plasma concentration) and cardiac muscle concentrations of the drug (up to 150 times plasma concentration), when compared to plasma concentration. ${ }^{14}$ Digibind (a digoxin-binding antibody) is indicated in cardiac arrest, when the plasma potassium levels exceed $5 \mathrm{mmol} / \mathrm{l}$, if there is a life-threatening dysrhythmia, when plasma digoxin levels exceed $12 \mathrm{ng} / \mathrm{ml}$, or if $>10 \mathrm{mg}$ (adult) or $>4 \mathrm{mg}$ (child) has been ingested. Treatment of toxicity includes gastrointestinal decontamination (acute toxicity), supportive treatment of hyper- or hypokalaemia, digoxin-binding antibodies (definitive treatment) and the management of unstable dysrhythmias as per advanced cardiac life support guidelines. ${ }^{13}$

Intravenous digoxin may only have limited applications, but it can be very useful in centres which deal with patients with complex comorbidities. Intravenous digoxin has also recently been removed from the Free State provincial formulary, indicating the loss of favour it has experienced in recent years. In the setting of patients presenting for urgent surgery with fast AF and COPD, however, this drug may avert catastrophic cardiac collapse due to bronchoconstriction and increased right ventricular afterload. Digoxin is currently advocated (together with beta blockers) for acute control of fast $A F$, where either drug is not contraindicated. ${ }^{1}$ Chronic oral digoxin therapy may also be considered for rate control in patients with heart failure or sedentary lifestyles, as digoxin is not as effective at limiting maximal heart rate during exertion, but assists with improving left ventricular systolic dysfunction. ${ }^{1,5}$ No mortality benefit to the use of digoxin (over other agents) can be identified except where no other treatment is instituted. ${ }^{15}$ These guidelines and suggestions may render digoxin a valuable and necessary tool in the modern anaesthetist's armamentarium and demand a thorough understanding of digoxin's pharmacodynamics and pharmacokinetics to allow for the appropriate use of digoxin in specialised circumstances.

\section{Acknowledgements}

The author would like to acknowledge Professor Johan Diedericks for always motivating him to participate in expanding the body of medical knowledge.

\section{Funding source}

The author did not receive any financial contribution towards the production of this article.

\section{Conflict of interest}

No conflicts of interest exist in the production of this article.

\section{Ethics approval}

Obtained from the University of the Free State's Ethics committee: UFS-HSD2019/1273/0110.

\section{ORCID}

R Swart (iD https://orcid.org/0000-0003-3873-4424

\section{References}

1. January CT, Wann LS, Alpert JS, et al. 2014 AHA/ACC/HRS Guideline for the management of patients with atrial bibrillation. J Am Coll Cardiol. 2014 Dec;64(21):e1-76. https://doi.org/10.1016/j.jacc.2014.03.022

2. McManus DD, Rienstra M, Benjamin EJ. An update on the prognosis of patients with atrial fibrillation. Circulation. 2012;126(10):e143-e146. https://doi. org/10.1161/CIRCULATIONAHA.112.129759.

3. Van Diepen S, Bakal JA, McAlister FA, Ezekowitz JA. Mortality and readmission of patients with heart failure, atrial fibrillation, or coronary artery disease undergoing noncardiac surgery: An analysis of 38047 patients. Circulation. 2011;124(3):289-96. https://doi.org/10.1161/CIRCULATIONAHA.110.011130.

4. Tabata T, Grimm RA, Asada J, et al. Determinants of LV diastolic function during atrial fibrillation: beat-to-beat analysis in acute dog experiments. Am J Physiol Circ Physiol. 2004 Jan;286(1):H145-52. https://doi.org/10.1152/ ajpheart.00588.2003.

5. Hack M, Flannery DJ, Schluchter $M$, et al. A comparison of rate control and rhythm control in patients with atrial fibrillation. N Engl J Med. 2002 Dec 5;347(23):1825-33. https://doi.org/10.1056/NEJMoa021328.

6. Khan IA, Nair CK, Singh N, Gowda RM, Nair RC. Acute ventricular rate control in atrial fibrillation and atrial flutter. Int J Cardiol. 2004;97(1):7-13. https://doi. org/10.1016/j.ijcard.2003.08.006.

7. Welch E, Milner A, editors. Applied Pharmacology in Anaesthesiology and Critical Care. 1st ed. Centurion: Medpharm Publications (PTY) LTD; 2012.

8. Yilmaz MB, Laribi $S$, Mebazaa A. Managing beta-blockers in acute heart failure: When to start and when to stop? Curr Heart Fail Rep. 2010;7(3):110-5. https://doi. org/10.1007/s11897-010-0014-8.

9. Virgadamo S. Digoxin: A systematic review in atrial fibrillation, congestive heart failure and post myocardial infarction. World J Cardiol. 2015;7(11):808-816. https://doi.org/10.4330/wjc.v7.i11.808.

10. Weinberger $\mathrm{MH}$. Antihypertensive therapy and lipids. Evidence, mechanisms, and implications. Arch Intern Med. 1985 Jun;145(6):1102-5.

11. Lei M, Wu L, Terrar DA, Huang CLH. Modernized classification of cardiac antiarrhythmic drugs. Circulation. 2018;138(17):1879-96. https://doi. org/10.1161/CIRCULATIONAHA.118.035455.

12. Grecu L. Autonomic. In: Barash PG, Cullen BF, Stoelting RK, Cahalan MK, Stock CM, Ortega R, editors. Clinical Anesthesia. 7th ed. Philadelphia: Wolters Kluwer Health; 2013. p. 395.

13. Nickson C. Digoxin Toxicity [Internet]. Life in the fast lane. 2019. p. 3. Available from: https://litfl.com/digoxin-toxicity-ccc/.

14. Worthley LI, Holt AW. Digoxin in the critically ill patient. Crit Care Resusc [Internet]. 1999;1(3):252-64. Available from: http://www.ncbi.nlm.nih.gov/ pubmed/16603014.

15. England TN. The effect of digoxin on mortality and morbidity in patients with heart failure. N Engl J Med. 1997 Feb 20;336(8):525-33. https://doi.org/10.1056/ NEJM199702203360801. 